\title{
Etiology, clinical characteristics, and outcomes of acute pancreatitis in patients at Assiut University Hospital
}

Enas Ahmed Reda Alkareemy, Lobna Abdel-Wahid Ahmed, Muhammad Abbas El-Masry, Hany Ayad Habib*iD and Mohamed H. Mustafa

\begin{abstract}
Background: Acute pancreatitis is one of the main causes of acute abdomen. It may cause multi-organ failure or even death. High morbidity and mortality are associated with it. The research study aimed at evaluating the clinical characteristics and results of acute pancreatitis in our community.

Results: Mean age of patients was $50.96 \pm 9.71$ years and 30 (60\%) patients were males. Gallstone was the most frequent etiology (56\%) followed by idiopathic pancreatitis (26\%). The majority of patients improved and only four patients died. Old age, presence of comorbidities, and leucocytosis were risk factors for a severe attack while old ages, presence of comorbidities, severe pancreatitis, and presence of complications were associated with mortality.

Conclusion: Acute pancreatitis could have serious outcomes if not correctly and early managed. It is recommended to perform multicenter studies with a large sample of patients. A multi-disciplinary team is required to assess idiopathic pancreatitis.
\end{abstract}

Keywords: Acute pancreatitis, Idiopathic pancreatitis, Ranson's criteria, Gallstones, Hypertriglyceridemia

\section{Background}

Acute pancreatitis is an inflammation of the pancreas with different organ involvement and with potentially devastating effects. Diagnosis of different grades of the disease can be overlooked and death can occur in $10 \%$ of patients with serious illness before diagnosis [1].

Generally, gallstones and alcohol abuse account for $70 \%$ of cases of acute pancreatitis. Gallstones, including microlithiasis, are the most common cause of acute pancreatitis which accounts for 35 to $40 \%$ of cases [2].

Hypertriglyceridemia causes about 2-5\% of cases of acute pancreatitis. The mechanism for hypertriglyceridemia to cause acute pancreatitis involves hydrolysis of triglycerides by pancreatic lipase which leads to the release of free fatty acids that damage pancreatic acinar cells and capillary endothelium [3].

* Correspondence: hanyayad1690@yahoo.com

Department of Internal Medicine, Faculty of Medicine, Assiut University, Assiut 71621, Egypt

\section{Springer Open}

Hypercalcemia may cause acute pancreatitis as a result of calcium deposition in the pancreatic duct and trypsinogen activation within the pancreatic parenchyma. Clinical characteristics and severity of acute pancreatitis with extrapancreatic organ failure are systemic inflammatory response syndrome (SIRS) elicited by lesion of the acinar cells [4].

Pancreatic auto-digestion is caused by the intra-pancreatic release of active pancreatic enzymes. This establishes a vicious cycle of active enzymes that damage acinar cells, vascular endothelium, and interstitium [4].

Pancreatitis warning signs include fever which suggests infection or inflammation, hypovolemia, Grey-Turner's sign and Cullen's sign that indicates hemorrhagic pancreatitis, tetany caused by hypocalcemia, and fulminant pancreatitis [5].

Fever is a critical sign of acute pancreatitis. It is mediated initially by inflammatory cytokines released in the 1st week. Fever may occur in the 2nd or 3rd week by

(c) The Author(s). 2020 Open Access This article is licensed under a Creative Commons Attribution 4.0 International License, which permits use, sharing, adaptation, distribution and reproduction in any medium or format, as long as you give appropriate credit to the original author(s) and the source, provide a link to the Creative Commons licence, and indicate if changes were made. The images or other third party material in this article are included in the article's Creative Commons licence, unless indicated otherwise in a credit line to the material. If material is not included in the article's Creative Commons licence and your intended use is not permitted by statutory regulation or exceeds the permitted use, you will need to obtain permission directly from the copyright holder. To view a copy of this licence, visit http://creativecommons.org/licenses/by/4.0/. 
infection of necrotic tissues. Also, pleural effusion may occur in some patients [5].

Acute pancreatitis diagnosis is based on standard abdominal pain, a 3-fold elevation in serum amylase or lipase level, and confirmatory results on cross-sectional abdominal imaging [6]. The current work was planned at evaluating the etiology, clinical characteristics, and outcomes of acute pancreatitis in our community.

\section{Methods}

The current study is a cross-sectional study that was performed in the period from January 2018 to December 2018.

Fifty patients with acute pancreatitis were enrolled in the study. It was diagnosed based on at least two criteria of the following: (1) typical abdominal pain, (2) elevated amylase and/or lipase greater than 3 times, and (3) radiological findings are matching with acute pancreatitis [6].

\section{Sample size calculation}

An estimated minimum sample size of 48 acute pancreatitis cases was needed to carry out the study according to the equation for the sample size of descriptive study design [7] using a prevalence of $2 \%$ [8], type I error of 0.05 , and power of $80 \%$.

Exclusion criteria included (1) age < 16 years, (2) patient with recurrent acute pancreatitis, and (3) patient with chronic pancreatitis.

All patients were subjected to thorough history taking and clinical evaluation. Liver function tests (LFTs), coagulation profile, kidney function tests (KFTs), complete blood count $(\mathrm{CBC})$, serum calcium level, serum amylase, serum lipase, lipid profile, $\mathrm{C}$-reactive protein (CRP), blood glucose level, and lactate dehydrogenase (LDH) were done.

Patients were initially evaluated with abdominal ultrasound and abdominal computed tomography (CT) was done if needed. Also, for each patient identified, the following data were assessed: demographic data, comorbidities, medications, laboratory results, imaging results, and outcomes.

\section{Statistical analysis}

Data were collected and analyzed using SPSS (Statistical Package for the Social Science, version 20, IBM, and Armonk, New York). Continuous data were expressed in form of mean \pm SD or median while nominal data were expressed in form of frequency (percentage).

$C h i^{2}$ test was used to compare the nominal data of different groups in the study while Student $t$ test was used to compare the mean of different two groups. Multivariate regression analysis was used to determine the independent risk factors for the prediction of severe pancreatitis and mortality in the current study. The level of confidence was kept at $95 \%$ and the $P$ value was significant if $<0.05$.

\section{Results}

Demographic and baseline laboratory data of studied patients (Table 1)

The mean age of patients was $50.96 \pm 9.71$ years with a range between 24 and 73 years. Out of the studied patients, $30(60 \%)$ patients were males and $20(40 \%)$ patients were females. Diabetes mellitus and hypertension presented in $15(30 \%)$ and $13(26 \%)$ patients, respectively. It was noticed that $11(22 \%)$ had more than one comorbidity.

Table 1 Demographic and baseline laboratory data

\begin{tabular}{ll}
\hline & $\boldsymbol{N}=\mathbf{5 0}$ \\
\hline Age (years) & $50.96 \pm 9.71$ \\
$\quad$ Range & $24-73$ \\
Sex & \\
Male & $30(60 \%)$ \\
Female & $20(40 \%)$
\end{tabular}

\section{Comorbidities}

Diabetes mellitus

Hypertension $13(26 \%)$

Ischemic heart disease $3(6 \%)$

Chronic kidney disease

$3(6 \%)$

Bronchial asthma

1 (2\%)

Multiple comorbidities

$11(22 \%)$

Smoking

$14(28 \%)$

Complete blood picture

Hemoglobin (g/dl)

$12.36 \pm 1.45$

Platelets $\left(10^{9} / \mathrm{I}\right)$

$256.30 \pm 43.34$

Leucocytic count $\left(10^{9} /\right.$ I)

$9.87 \pm 3.67$

Liver function tests

Bilirubin (mg/dl)

$2.79 \pm 1.01$

Direct bilirubin (mg/dl)

$0.98 \pm 0.23$

Aspartate transaminase (U/L)

$26.92 \pm 10.78$

Alanine transaminase (U/L)

$33.97 \pm 4.46$

Alkaline phosphatase (U/L)

$155.49 \pm 39.38$

Albumin (mg/dl)

$33.23 \pm 3.94$

Kidney function tests

Urea $(\mathrm{mmol} / \mathrm{l})$

$9.89 \pm 2.78$

Creatinine $(\mu \mathrm{mol} / \mathrm{l})$

$128.98 \pm 34.09$

Serum electrolytes

Calcium (mg/dl)

$8.99 \pm 1.89$

Sodium $(\mu \mathrm{mol} / \mathrm{l})$

$137.87 \pm 3.98$

Potassium ( $\mu \mathrm{mol} / \mathrm{l})$

$4.44 \pm 1.01$

Random blood sugar (mg/dl)

$137.09 \pm 19.87$

Serum amylase $(U / L)$

$789.98 \pm 333.01$

Serum lipase $(U / L)$

$687.01 \pm 230.54$

Data were expressed in form of mean (SD) and range (frequency) 
Table 2 Etiology of acute pancreatitis in studied patients

\begin{tabular}{llll}
\hline Etiology & $\boldsymbol{N}=\mathbf{5 0}$ & Males $(\boldsymbol{n}=\mathbf{3 0})$ & Females $(\boldsymbol{n}=\mathbf{2 0})$ \\
\hline Gallstones & $28(56 \%)$ & $13(43.3 \%)$ & $15(75 \%)$ \\
Idiopathic & $13(26 \%)$ & $11(36.7 \%)$ & $2(10 \%)$ \\
Post-ERCP & $6(12 \%)$ & $4(13.3 \%)$ & $2(10 \%)$ \\
Hypertriglyceridemia & $3(6 \%)$ & $2(6.7 \%)$ & $1(5 \%)$ \\
\hline
\end{tabular}

Data were expressed in form of frequency (percentage)

Thirteen (26\%) patients had leucocytosis. Serum electrolytes including sodium, potassium, and calcium were normal in studied patients. Other data are summarized in Table 1

Etiology of acute pancreatitis in studied patients (Table 2) Twenty-eight patients (56\%) had acute pancreatitis secondary to gall bladder stones, and 13 patients $(26 \%)$ and 6 patients (12\%) had idiopathic and post-endoscopic retrograde cholangiopancreatography pancreatitis (ERCP), respectively. Only in three patients (6\%), the etiology was attributed to hypertriglyceridemia.

\section{The severity of pancreatitis based on Ranson's criteria} and CT findings (Fig. 1)

Based on Ranson's criteria, 30 (60\%) patients had mild pancreatitis and 9 (18\%) patients had moderate pancreatitis while $25(50 \%)$ and $14(28 \%)$ patients had mild and moderate pancreatitis respectively based on abdominal CT findings. It was noticed that 11 (22\%) patients had severe pancreatitis based on either CT findings or Ranson's criteria.
Hospital stay and outcome in studied patients (Table 3,

Fig. 2)

The mean hospital stay in studied patients was $6.89 \pm$ 1.98 days. The majority (92\%) of patients improved while 4 patients (8\%) died. As regarding complications, 36 (72\%) patients had no complications. Each of respiratory failure, renal failure, paralytic ileus, pancreatic abscess, and diabetes mellitus occurred in 4 (8\%), $3(6 \%), 3$ (6\%), $1(2 \%)$, and $1(2 \%)$ patients, respectively.

Two patients developed multi-organ failure and died. Also, two patients died secondary to respiratory failure. Regarding the etiology of pancreatitis in the dead patients; two patients had gallstones, one patient had hypertriglyceridemia, and one patient had idiopathic pancreatitis.

\section{Predictors of severe acute pancreatitis and mortality in the current study (Tables 4 and 5)}

The predictors for severe pancreatitis were old age, presence of comorbidities and leucocytosis while predictors for mortality among patients with acute pancreatitis were old age, presence of comorbidities, severe pancreatitis and presence of complications.

\section{Discussion}

There are many causes of acute pancreatitis which in $75-85 \%$ of patients can be identified. The most frequent causes of acute pancreatitis are obstruction of the common bile duct by stones (38\%) and alcohol abuse (36\%) in developed countries. Pancreatitis triggered by gallstone is caused by gallstone movement in the duct. The blockage is located in the bile duct or pancreatic duct, or both [9].

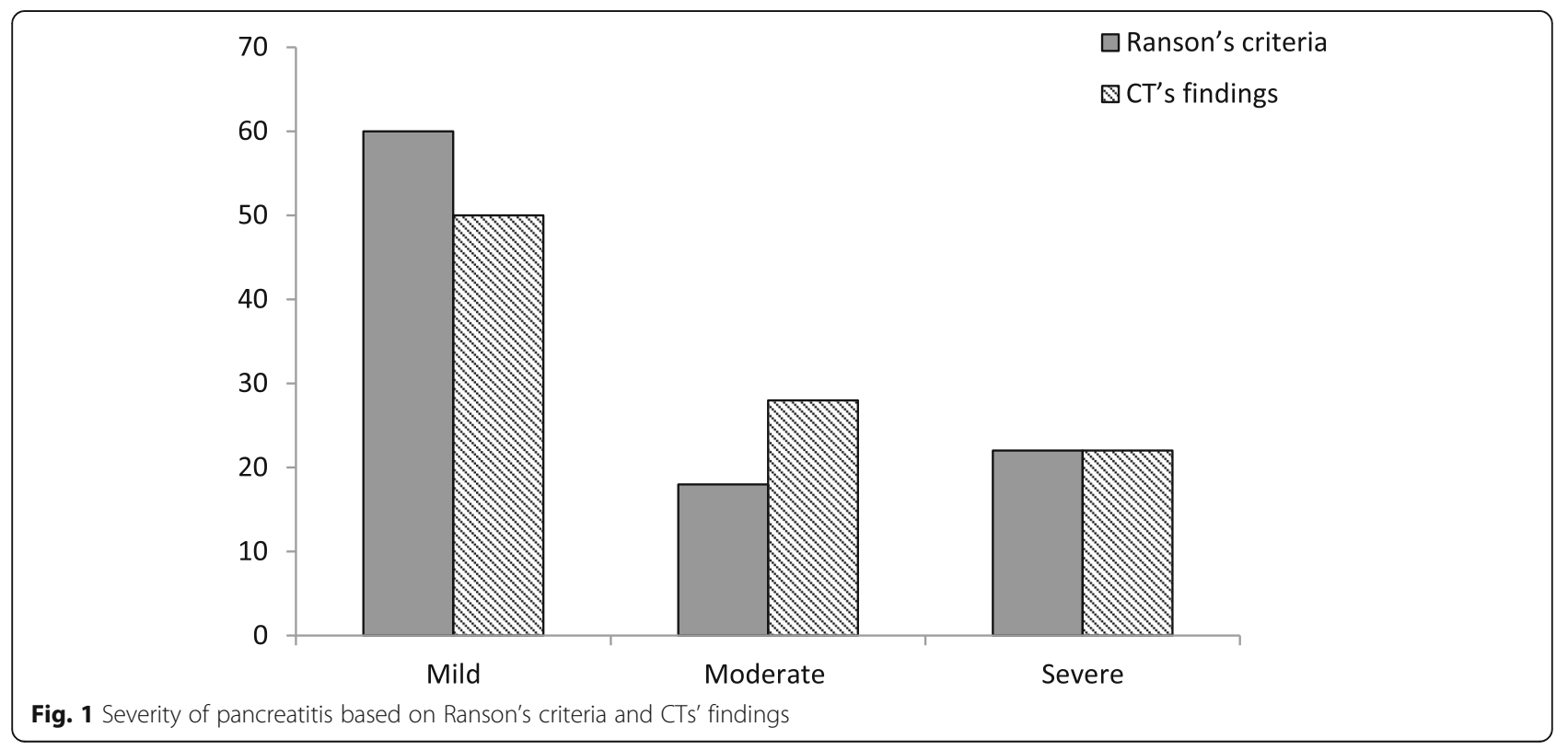


Table 3 Hospital stay and outcome in studied patients

\begin{tabular}{ll}
\hline Hospital stay (day) & $\boldsymbol{N}=\mathbf{5 0}$ \\
Complications & $6.89 \pm 1.98$ \\
Nothing & \\
Respiratory failure & $36(72 \%)$ \\
Renal failure & $4(8 \%)$ \\
Paralytic ileus & $3(6 \%)$ \\
Pancreatic abscess & $3(6 \%)$ \\
Diabetes mellitus & $1(2 \%)$ \\
Multi-organ failure & $1(2 \%)$ \\
Outcome & $2(4 \%)$ \\
Survivors & \\
Non-survivors & $46(92 \%)$ \\
Respiratory failure & $4(8 \%)$ \\
Multi-organ failure & $2(4 \%)$ \\
\hline
\end{tabular}

Data were expressed in form of mean (SD) and frequency (percentage)

The current study included 50 patients who were diagnosed to have acute pancreatitis aiming to assess different etiologies, clinical characteristics, and outcomes of acute pancreatitis. The mean age of those patients was $50.96 \pm 9.71$ years with a range between 24 and 73 years. Thirty (60\%) patients were males and $20(40 \%)$ patients were females. In agreement with our study, Nesvaderani et al. observed that the mean age of their patients was 50 years $[10]$.

In contrast to the current study, Vengadakrishnan and Koushik in their study observed that most patients were in the age group of 21 to 40 years; also, mean age of patients was lower as reported by Ekka et al. that showed a total of $38(40 \%)$ patients was in the 4th decade of their life with a mean age of 36.14 years $[11,12]$.
The current study included 50 patients, $30(60 \%)$ were males and 20 (40\%) were females; this agreed with multiple studies such as Vengadakrishnan and Koushik and Lindkvist et al. that showed a higher incidence of acute pancreatitis in males $[11,13]$.

In contrast to the current study, Nesvaderani et al. published their retrospective study of 932 patients and observed that $50.4 \%$ of patients were females while $49.6 \%$ were males [10].

The most frequent etiology in the current study was gallstones 28 (56\%) patients. The etiology was idiopathic in $13(26 \%)$ patients. It was noticed that acute pancreatitis occurred secondary to ERCP in $6(12 \%)$ patients and hypertriglyceridemia in $3(6 \%)$ patients.

Baig et al. observed alcoholism was the most frequent cause followed by biliary stones while Wang et al. confirmed that biliary stones (38\%) and alcohol abuse (36\%) were the most frequent causes of acute pancreatitis $[14,15]$.

Acute pancreatitis causes differ according to the area studied worldwide. Large Ireland and America retrospective studies showed a 1:1 ratio of alcohol and gallstone pancreatitis with each cause contributing $23-36 \%$ in these areas. A European study found that gallstones clearly predominated over alcohol in southern European countries, but a similar frequency in northern European countries, which was due to differences in alcohol consumption in different regions $[16,17]$.

It is known that hormonal influence, particularly estrogen, plays a major role in the formation of gallstones in females and increases the risk for pancreatitis. Then, it was inspected on whether gender had any role in the severity of the disease, but there was no significant difference with $P$ value $>0.05$ and this was similar to a previous study published by Lankisch et al. Besides, the

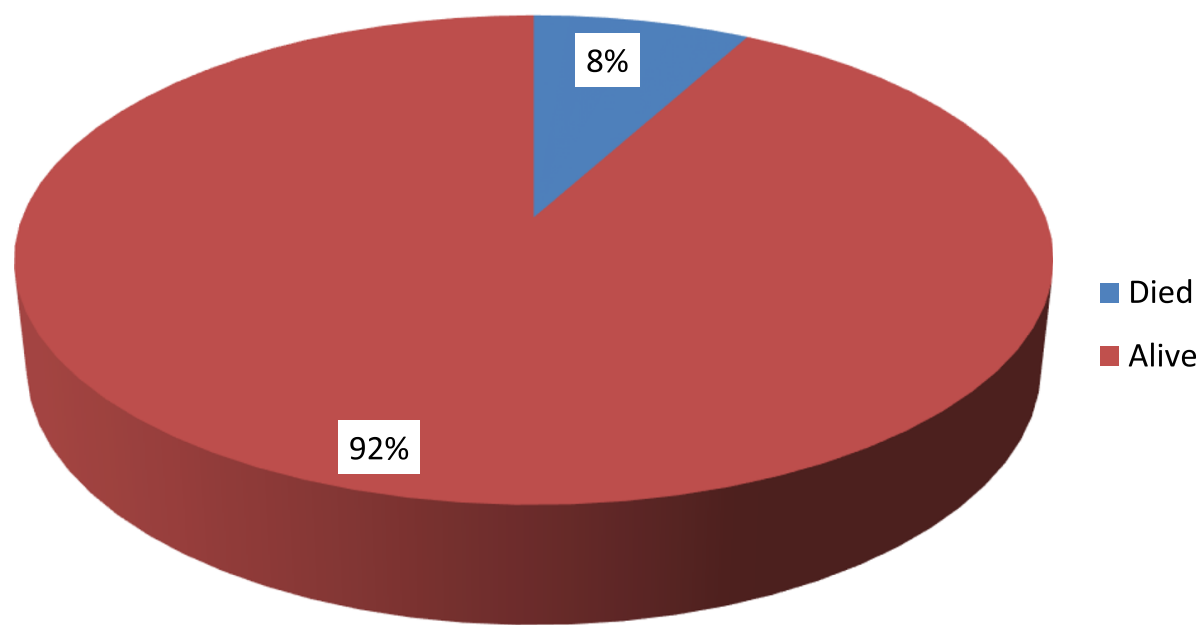

Fig. 2 Outcome of patients in the current study 
Table 4 Predictors for severe acute pancreatitis in the current study

\begin{tabular}{llll}
\hline Variables & Odds ratio & $\mathbf{9 5 \%}$ confidence interval & $P$ value \\
\hline Old age & 2.87 & $1.53-4.44$ & 0.03 \\
Male sex & 0.98 & $0.67-1.02$ & 0.45 \\
Presence of comorbidities & 3.33 & $1.65-5.87$ & 0.01 \\
Diabetes mellitus & 1.01 & $0.38-1.98$ & 0.67 \\
Hypertension & 1.11 & $0.33-3.01$ & 0.19 \\
Leucocytosis & 2.04 & $1.11-4.56$ & 0.03 \\
Etiology & 1.54 & $0.56-3.45$ & 0.60 \\
\hline
\end{tabular}

$P$ value was significant if $<0.05$

causes of acute pancreatitis did not influence the severity of the disease [18].

Based on Ranson's criteria, 30 (60\%) patients had mild pancreatitis and $9(18 \%)$ patients had moderate pancreatitis, while $25(50 \%)$ and 14 (28\%) patients had mild and moderate pancreatitis, respectively, based on abdominal CT findings. It was noticed that $11(22 \%)$ patients had severe pancreatitis based on either CT findings or Ranson's criteria.

It was found that 11 out of the 13 severe cases were correctly labeled by abdominal CT while 16 out of 22 moderate cases and 30 out of 47 mild cases. This obviously showed that the use of CT is more indicated in the severe cases and its use is not recommended in the mild and moderate cases, not only because of its lower sensitivity but also to avoid unnecessary radiation [19].

In this study, the mean hospital stay in studied patients was $6.89 \pm 1.98$ days ranging from 3 to 10 days. Most of the patients (92\%) had improved. Regarding complications of acute pancreatitis in studied patients, 36 (72\%) patients had no complications. Each of respiratory failure, renal failure, paralytic ileus, pancreatic abscess, diabetes mellitus, and multi-organ failure occurred in $4(8 \%), 3(6 \%), 3(6 \%), 1(2 \%), 1(2 \%)$, and $2(4 \%)$ patients, respectively.

The predictors of severe pancreatitis were old age, presence of comorbidities, and leucocytosis while predictors of mortality among patients with acute pancreatitis were old age, presence of comorbidities, severe pancreatitis, and presence of complications.

Albulushi et al. found that 9 out of the 14 severe cases experienced complications (64\%) compared to $25 \%$ in mild cases and $48 \%$ in the moderate cases. This sequence is logical in the term that patients with severe acute pancreatitis had more morbidities compared to mild and moderate cases [19].

Current guidelines suggest that mortality from acute pancreatitis should be less than $10 \%$ overall. The mortality rate in this study was $8 \%$. It was reported that a lower than rates of $1.5-4.2 \%$ reported in large epidemiological studies of pancreatitis in other countries [20].

\section{Conclusion}

The current study included 50 patients with acute pancreatitis. The mean age of those patients was $50.96 \pm$ 9.71 years. The study included 30 male patients and 20 female patients. The most frequent etiology in the current study was gallstones which cause acute pancreatitis in 28 patients (56\%). The etiology was idiopathic in $13(26 \%)$ patients. It was noticed that acute pancreatitis occurred secondary to ERCP in $6(12 \%)$ patients and hypertriglyceridemia in $3(6 \%)$ patients.

The majority of patients improved and only four patients died. Old age, presence of comorbidities, and

Table 5 Predictors for mortality in the current study

\begin{tabular}{|c|c|c|c|}
\hline Variables & Odds ratio & $95 \%$ confidence interval & $P$ value \\
\hline Old age & 2.11 & $1.98-5.01$ & 0.04 \\
\hline Male sex & 0.98 & $0.67-1.02$ & 0.45 \\
\hline Presence of comorbidities & 2.02 & $1.21-3.08$ & 0.01 \\
\hline Diabetes mellitus & 1.01 & $0.38-1.98$ & 0.67 \\
\hline Hypertension & 1.11 & $0.33-3.01$ & 0.19 \\
\hline Leucocytosis & 2.04 & $1.11-4.56$ & 0.03 \\
\hline Etiology & 1.54 & $0.56-3.45$ & 0.60 \\
\hline Severe pancreatitis & 2.46 & $1.98-4.09$ & 0.02 \\
\hline Presence of complication & 4.38 & $2.22-9.09$ & 0.01 \\
\hline
\end{tabular}

$P$ value was significant if $<0.05$ 
leucocytosis were risk factors for a severe attack while old ages, presence of comorbidities, severe pancreatitis, and presence of complications were associated with mortality.

\section{Recommendations}

Routine investigations as LFTs, KFTs, CBC, serum calcium, LDH, amylase, lipase, and blood glucose should be evaluated at the time of admission for any patient suspected to have acute pancreatitis for early diagnosis, severity scoring, and management of acute pancreatitis which will affect the outcomes.

Treatable causes of acute pancreatitis should be identified and managed appropriately to prevent the recurrence of acute pancreatitis.

Our study recommends further studies that include more patients and more clinical and laboratory methods for early diagnosis and detection of the causes of acute pancreatitis and the outcomes.

\section{Abbreviations}

CBC: Complete blood count; CRP: C-reactive protein; CT: Computed tomography; ERCP: Endoscopic retrograde cholangiopancreatography; KFTs: Kidney function tests; LDH: Lactate dehydrogenase; LFTs: Liver function tests; SIRS: Systemic inflammatory response syndrome

\section{Acknowledgements}

Not applicable

\section{Authors' contributions}

EA: contributed to study design, manuscript writing, and revision. LA: contributed to study design, data collection and analysis, and manuscript preparation. MA: substantial contributions to the conception of the work. MH: acquisition, analysis, and interpretation of data. HA: acquisition, analysis, and interpretation of data. All authors have read and approved the manuscript.

\section{Funding}

Not applicable

\section{Availability of data and materials}

The datasets used and/or analyzed during the current study are available from the corresponding author on reasonable request.

\section{Ethics approval and consent to participate}

There were no risks during the application of the research.

Privacy and confidentiality were maintained during all stages of assessment Every patient subjected to this study was informed about the results of the research.

The work was approved by the Ethical Committee of faculty of medicine of Assiut University (approval number 17101193).

Consent (verbal) was obtained from the patient to participate in this study. Refusal would not affect medical services which are usually offered. Verbal consent was approved by the ethics committee.

\section{Consent for publication}

Consent for publication (verbal) was obtained from all the participating patients. Verbal consent was approved by the ethics committee.
Received: 10 September 2020 Accepted: 22 October 2020

Published online: 04 November 2020

\section{References}

1. Kapoor S, Singh D, Chander R (2018) To study the utility of Modified Glasgow Score and CT Severity Score in acute pancreatitis-a clinical study. J Adv Med Dent Sci Res 6:114-117

2. Negi N, Mokta J, Sharma B, Sharma R, Jhobta A, Bodh V, Ranjan A (2018) Clinical profile and outcome of acute pancreatitis: a hospital-based prospective observational study in Subhimalayan State. J Assoc Physicians India 66:22-24

3. Murphy MJ, Sheng X, MacDonald T, Wei L (2012) Hypertriglyceridemia and acute pancreatitis. Arch Intern Med 26:1-3

4. Trezevant MS, Winton JC, Holmes AK (2019) Hypercalcemia-induced pancreatitis in pregnancy following calcium carbonate ingestion. J Pharm Pract 32:225-227

5. Rao BS, Sree Vane M, Chandra VS (2014) Etiology, clinical profile, severity and outcome of acute pancreatitis in relation to bedside index for severity of acute pancreatitis BISAP and CT severity index [CTSI] scores. Int J Med Res Health Sci 3:922-928

6. Orkin SH, Trout AT, Fei L, Lin TK, Nathan JD, Thompson T, Vitale DS (2019) Sensitivity of biochemical and imaging findings for the diagnosis of acute pancreatitis in children. J Pediatr 213:143-148 e142

7. Wingo P, Phyllis A, Higgins JE, Rubin GL, Zahniser SC et al (1994) An epidemiological approach to reproductive health. Geneva, p. 15-67

8. Kirkegård J, Cronin-Fenton D, Heide-Jørgensen U, Mortensen FV (2018) Acute pancreatitis and pancreatic cancer risk: a nationwide matched-cohort study in Denmark. Gastroenterology 154(6):1729-1736

9. Lankisch PG, Assmus C, Lehnick D, Maisonneuve P, Lowenfels AB (2001) Acute pancreatitis: does gender matter? Dig Dis Sci 46:2470-2474

10. Nesvaderani M, Eslick GD, Vagg D, Faraj S, Cox MR (2015) Epidemiology, etiology and outcomes of acute pancreatitis: a retrospective cohort study. Int J Surg 23:68-74

11. Vengadakrishnan K, Koushik A (2015) A study of the clinical profile of acute pancreatitis and its correlation with severity indices. Int J Health Sci 9:410

12. Ekka NMP, Mishra G, Kumar V, Tiwary AK, Kar T, Tiwary A (2018) Clinical pattern of acute pancreatitis in eastern India and comparison of Ranson, BISAP and APACHE II as a predictor of severity, local complications and mortality. Int Surg J 5:3707-3712

13. Lindkvist B et al.(2008) Trends in incidence of acute pancreatitisin a Swedish population: is there really an increase? Clin Gastroenterol Hepatol x:2:831-837.

14. Baig SJ, Rahed A, Sen S (2008) A prospective study of the aetiology, severity and outcome of acute pancreatitis in Eastern India. Trop Gastroenterol 29:20

15. Wang X, Xu Y, Qiao Y, Pang X, Hong L, Fu J, Li Y (2013) An evidence-based proposal for predicting organ failure in severe acute pancreatitis. Pancreas 42:1255-1261

16. Gullo L, Migliori M, Oláh A, Farkas G, Levy P, Arvanitakis C, Lankisch P (2002) Acute pancreatitis in five European countries: etiology and mortality. Pancreas 24:223-227

17. O'Farrell A, Allwright S, Toomey D, Bedford D, Conlon K (2007) Hospital admission for acute pancreatitis in the Irish population, 1997-2004: could the increase be due to an increase in alcohol-related pancreatitis? J Public Health 29:398-404

18. Lankisch PG, Weber-Dany B, Hebel K, Maisonneuve P, Lowenfels AB (2009) The harmless acute pancreatitis score: a clinical algorithm for rapid initial stratification of nonsevere disease. Clin Gastroenterol Hepatol 7:702-705

19. Albulushi A, Siddiqi A, Alqarshoubi I, Aladawi M, Alkhadhouri G, Farhan H (2014) Pattern of acute pancreatitis in a tertiary care center in Oman. Oman Med J 29:358

20. Chen Y, Zak Y, Hernandez-Boussard PW, Visser BC (2013) The epidemiology of idiopathic acute pancreatitis, analysis of the nationwide inpatient sample from 1998 to 2007. Pancreas 42(1):1e5

\section{Publisher's Note}

Springer Nature remains neutral with regard to jurisdictional claims in published maps and institutional affiliations. 